\title{
Training general surgery residents on the gastroenterology service: A look at the successes and challenges
}

\author{
Susan Reid MD1, Stephen Vanner MD²
}

$\mathrm{D}^{\prime}$ Susan Reid is an associate professor in the Department of Surgery, McMaster University, Hamilton, Ontario, practicing general surgery and critical care. She is the Program Director of the General Surgery Residency Program and Chair of the Canadian Association of General Surgeons Postgraduate Education Committee.

General surgery (GS) resident rotations in gastroenterology (GI) training programs have traditionally been a regular component of GS training in Canada. These rotations have provided increased exposure to basic and clinical digestive sciences for the medical management of gastrointestinal disorders, and often provide the majority of the cognitive and practical endoscopic training. Recently, however, this endoscopic training has received increased scrutiny because of growing time constraints on endoscopy suites and the recognition that procedures conducted with trainees require significantly more time to complete. This issue is also coupled with the growing discussion regarding the definition of endoscopic competency, which could be heightened by the possibility of decreasing access to this training opportunity. In the present interview with Dr Susan Reid, Chair of the Surgical Residency Training Programs, I discuss the current value of this rotation to surgical training programs and address some future challenges.

SV: How many of the GS training programs rotate their residents through the GI training programs?

SR: Almost all GS training programs in Canada rotate their residents through medical GI training programs. However, in British Columbia, the GS program pulled the residents out of the GI medicine rotations because the low number of procedures performed was believed to be inadequate for training. This was an amicable separation, discussed with the GI service at the time. The substitute rotation is with general surgeons in two community sites where there is a high volume of endoscopy procedures performed. Based on supportive case logs and resident feedback, this rotation has been working well for the program. The program director in British Columbia has again initiated contact with the GI service and they are reviewing the possibility of a more collaborative approach. In Calgary, Alberta, the approach is a mix - the residents are placed on a two-month GI medicine service, but they also receive experience in the GS rotations, particularly in the community GS rotations. The serious limitation in Calgary is the restriction of upper endoscopy privileges for general surgeons and the limitation of colonoscopy privileges to colorectal surgeons.

SV: How long are these rotations and what is the perceived ideal length? What proportion of their endoscopic training do they receive during these rotations?

SR: The rotations vary from two months to three months in duration. Approximately 50\% of the rotation is spent in direct endoscopic training. The rotations occur within the postgraduate year (PGY) 2 or PGY 3. Further endoscopy training occurs in the context of GS rotations.

SV: What advantages do the surgical training programs perceive in having their trainees rotate through these programs? What are the components of these rotations apart from endoscopic training (ie, inflammatory bowel disease [IBD] clinics, liver disease, motility clinics, consults, ward coverage, gastrointestinal bleeds and pathology rounds)?

SR: The residents partake in inpatient and emergency room consultations, as well as ward patient care. They attend GI clinics that range from general GI to subspecialty clinics in IBD or liver disease. With the exception of one program, the residents do not typically attend motility clinics. The dedicated rotation usually occurs in the PGY 2 or PGY 3. This allows the resident to develop basic endoscopy skills that will be built on with further endoscopy experience on the GS rotations. During the dedicated two- to three-month rotation, the resident is expected to consolidate medical knowledge and clinical skills in the areas of gastrointestinal medicine that are particularly relevant to GS such as IBD, liver disease, gastrointestinal bleeding and malignant disease of the gastrointestinal tract. It is also expected that a good understanding of gastrointestinal physiology will be obtained.

SV: What do the surgical programs believe their trainees add to the GI programs?

SR: The GS residents work with the GI fellows on the same rotations. This allows the GI fellows and the GS resident to work together in a collaborative fashion, which includes the

${ }^{1}$ McMaster University, Hamilton; ${ }^{2}$ Queen's University, Kingston, Ontario

Correspondence: Dr Susan Reid, McMaster University Medical Centre, Room 2F, 1280 Main Street West, Hamilton,

Ontario L8S 4L8. Telephone 905-521-2617, fax 905-527-1700, e-mail reid@mcmaster.ca

Received for publication April 2, 2008. Accepted April 3, 2008 
exchange of perspectives. There are sites where the GS resident may be the only resident on the GI service; hence, the majority of the consultative service is provided by the surgical resident. This is certainly of benefit to the GI service and staff. In addition, the PGY 3 resident is senior enough to provide surgical input to the GI service if requested.

SV: What are the barriers to programs who want their trainees to rotate through GI training programs?

SR: Although most sites report no barriers to GI rotations, the number of residents in both the GI fellowship and the GS residency has increased; therefore, scheduling is occasionally a challenge. Assuming the volume of ward work and consultations is adequate, some sites do not have enough endoscopy time to support more than two residents per rotation. The gastroenterologists have been very receptive and supportive of the GS residents and our training programs. There are sites that have restricted access to endoscopy time for general surgeons, and this limits the ability of the program to provide endoscopy training in the senior years of training. To reduce the congestion in some programs, attempts have been made to initiate contacts with community gastroenterologists. So far, this has not yielded any new sites or clinical supervisors.

SV: Do any of the GI fellows rotate through surgical training programs? Should they?

SR: Currently, in most programs, the GI fellows do not spend any time in GS rotations. Most programs have offered this as an elective experience, but to date, this is routinely occurring only in a small number of centres. There would certainly be considerable benefit, especially in the subspecialties of colorectal surgery, upper gastrointestinal surgery and hepatobiliarypancreatic services. Most centres are also open to the GI fellow attending to the operating room for cases that they might have been involved with, such as gastrointestinal bleeds or toxic colitis that proceeds to surgical intervention. In some centres, the hepatobiliary surgeon is performing the majority of the endoscopic retrograde cholangiopancreatography (ERCP) procedures and there is opportunity for GI fellows to participate in this experience.

SV: What is the content of the endoscopic training that the surgical residents receive? Given that more general surgeons are increasing their endoscopy time in the communities and in smaller communities where they may be the only available endoscopist, is there a desire for trainees to be skilled in therapeutic endoscopy? How many surgical trainees want to acquire skills in ERCP during their core training?

SR: Through both the GI rotation and the academic half-day, the residents receive lectures on diseases of the entire gastrointestinal tract, as well as teaching sessions on endoscopy and therapeutic endoscopy. The GI staff provides these teaching sessions. There is a strong desire for residents to be trained in advanced endoscopy, especially in the realm of managing upper gastrointestinal bleeding, including variceal hemorrhage. The GS residents may be exposed to ERCP during core training, but are not at all expected to be proficient in ERCP. Those who are interested in further fellowship training are encouraged to undergo ERCP training in the context of a hepatobiliarypancreatic fellowship.

SV: A number of organizations including the The American Society for Gastrointestinal Endoscopy, The American Gastroenterological Association and the Canadian Association of Gastroenterology, have recommended minimum numbers of procedures before competency can be assessed. Do surgical training programs follow one of these guidelines or 'in-house' guidelines? Is this an issue for surgical training programs?

SR: The evaluation of a technical skill in GS is a component of the medical expert competency that has been well ingrained into the hearts and minds of general surgeons long before the Canadian Medical Education Directives for Specialists. Since the beginning of formal surgical training programs in North America, championed by Dr William Halsted, the mentoring, teaching, guiding and development of surgical skills in trainees has progressed from individual apprenticeships to what we know today as postgraduate training. This has assured that trainees are properly educated in all aspects of surgical care, with the knowledge base to support the use of technical and surgical interventions. Thus, it is imperative that a numberbased approach to competency is recognized as an inadequate method of evaluation. A trainee must have the ability to physically perform a procedure in a safe, efficient and effective manner, but must also know the indications, contraindications, outcomes, side effects/complications and alternatives, and how to communicate this holistic approach to colleagues and patients. In this manner, endoscopy is no different than any other surgical skill. There is no doubt that surgical trainees, compared with trainees in medical programs, will have a high level of basic technical proficiency, hand-eye coordination and the ability to transfer surgical skills to new procedures, allowing most trainees to develop endoscopy skills quickly. Hence, endoscopic skills are evaluated both inside and outside the endoscopy suite. Within the endoscopy suite, skills are evaluated via direct observation from the supervisors, with residents given increasing independence as demonstrated skills are deemed adequate. Most surgical residents receive a GI rotation early in training; then, further skill development is incorporated in GS rotations with the same direct observation as the process for competency evaluation. Formal written evaluations are completed for all rotations that include procedural evaluations. I believe this is the same process for the evaluation of endoscopy skills in medical GI residents. All that said, it is now mandatory that surgical residents track all procedures in an electronic database, and most of the Canadian training programs are using the same logging program. There has yet to be any concerns raised that Canadian GS residents have not been able to meet the number of requirements set out by the Acceditation Council for Graduate Medical Education; in fact, most residents will far surpass those numbers.

Training in endoscopic skills and the ongoing maintenance of those skills is imperative to the practice of GS in Canada. Our GS training programs are currently mandated by the Royal College of Physicians and Surgeons of Canada to provide adequate training to allow surgeons to incorporate endoscopy into their practices. There is absolutely no doubt that endoscopy will continue to be a necessary component of practice of the majority of general surgeons in Canada. As a group, the GS program directors in Canada are committed to working closely with our GI colleagues, and GI training programs to continue to provide excellent training for all residents. I am certain that the volume of patients requiring endoscopic procedures will continue to increase as our population increases, and the continued involvement of surgeons will be absolutely key to meeting 
those increased demands. The situation of restriction of privileges in Calgary is disturbing. This issue will be brought forward to both the Canadian Association of Gastroenterology's executive committee and the Royal College of Physicians and Surgeons of Canada.

$\mathrm{SV}$ : There is a great deal of educational value to be gained through interaction between the surgical and GI trainees and their teachers during their joint involvement in patient care. Does this occur through formal joint rounds at most centres or does it occur on an ad hoc basis during informal 'hall side' exchanges?

SR: There are several avenues for exchange in every training centre. The formal events include weekly GI and radiology rounds that are attended by GS, GI and drug information residents. Many centres have combined weekly IBD rounds, GI oncology rounds, tumour boards, GI/GS rounds and hepatobiliary rounds that are attended by GI and GS residents and staff. In Hamilton, a 'gastroenterology supper club' meets every second month and is attended by academic and community gastroenterologists, surgeons and residents. In Hamilton, the GI service is centered at one site, and the ward is shared by GS and medical GI. The academic GS offices are in the same location as the academic GI offices, and GS and GI share clinic space and endoscopy space. There is a similar arrangement in Calgary. This allows for improved communication and facilitates informal exchanges. 


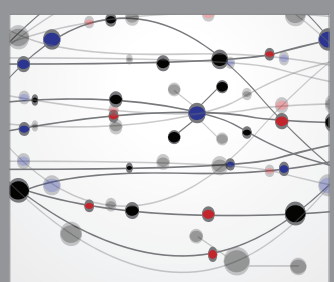

The Scientific World Journal
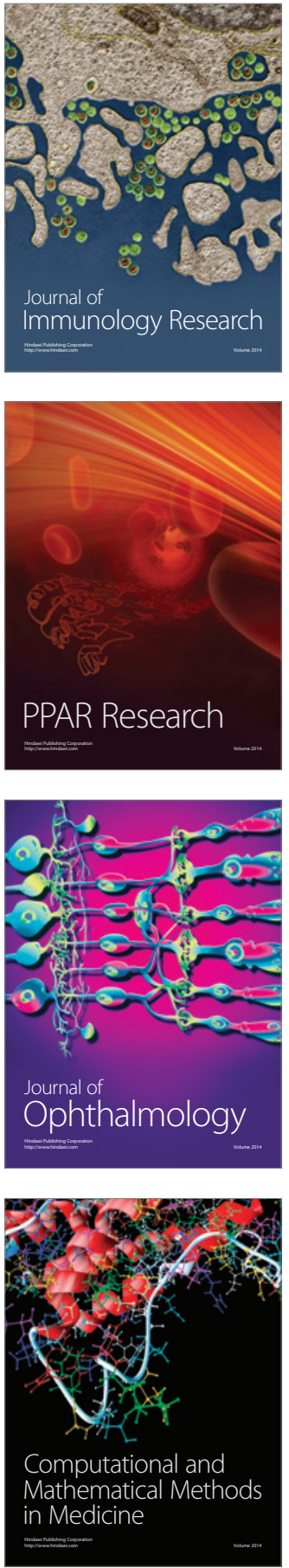

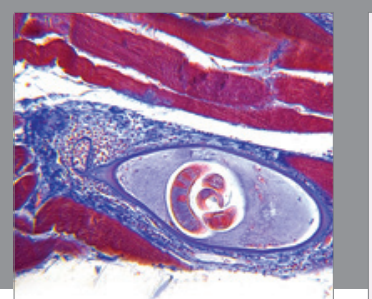

Gastroenterology Research and Practice

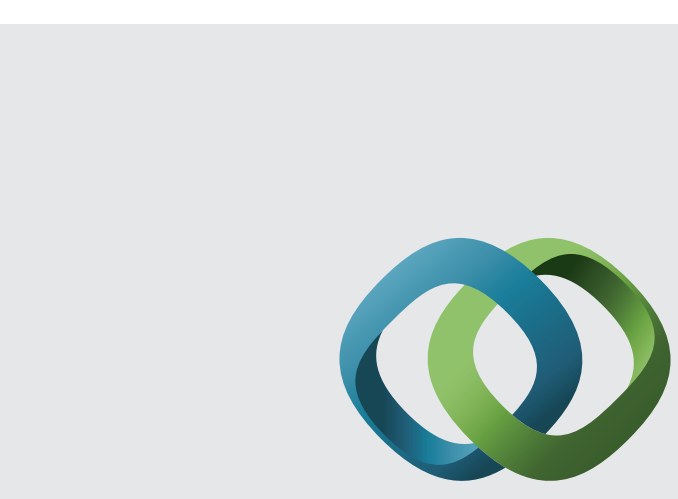

\section{Hindawi}

Submit your manuscripts at

http://www.hindawi.com
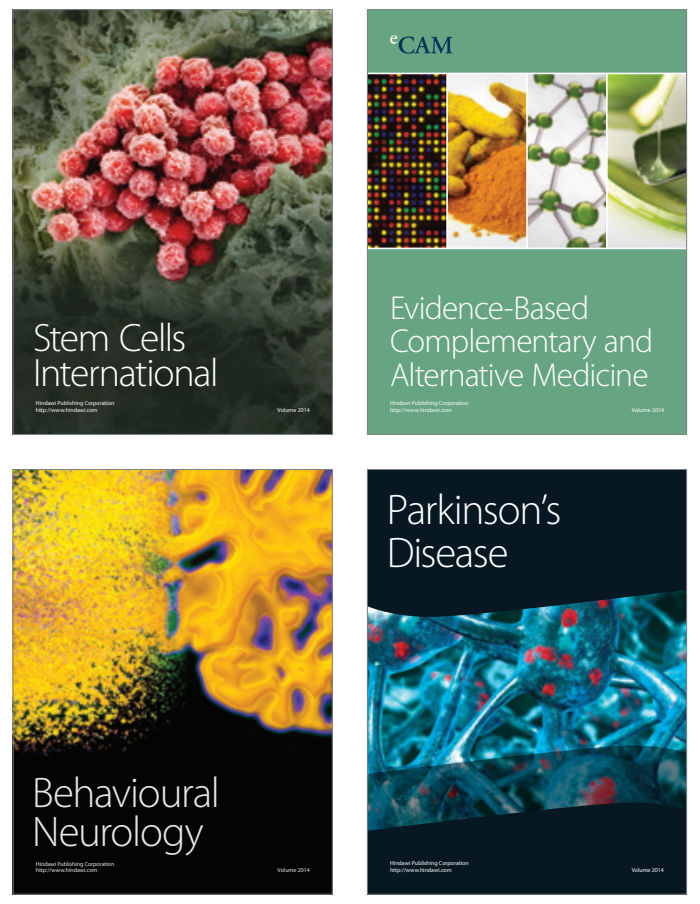
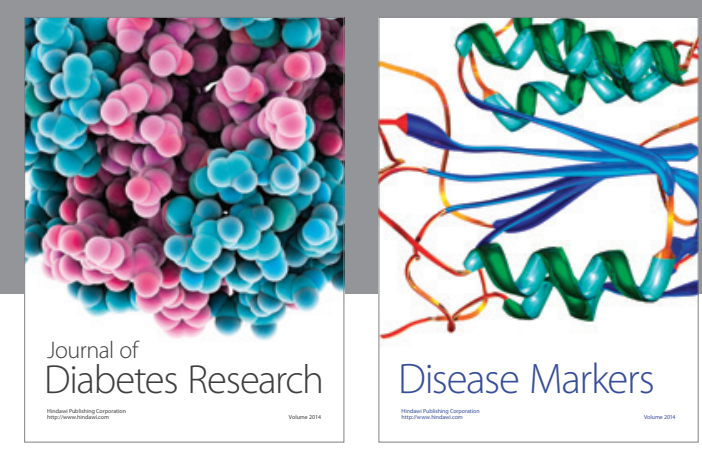

Disease Markers
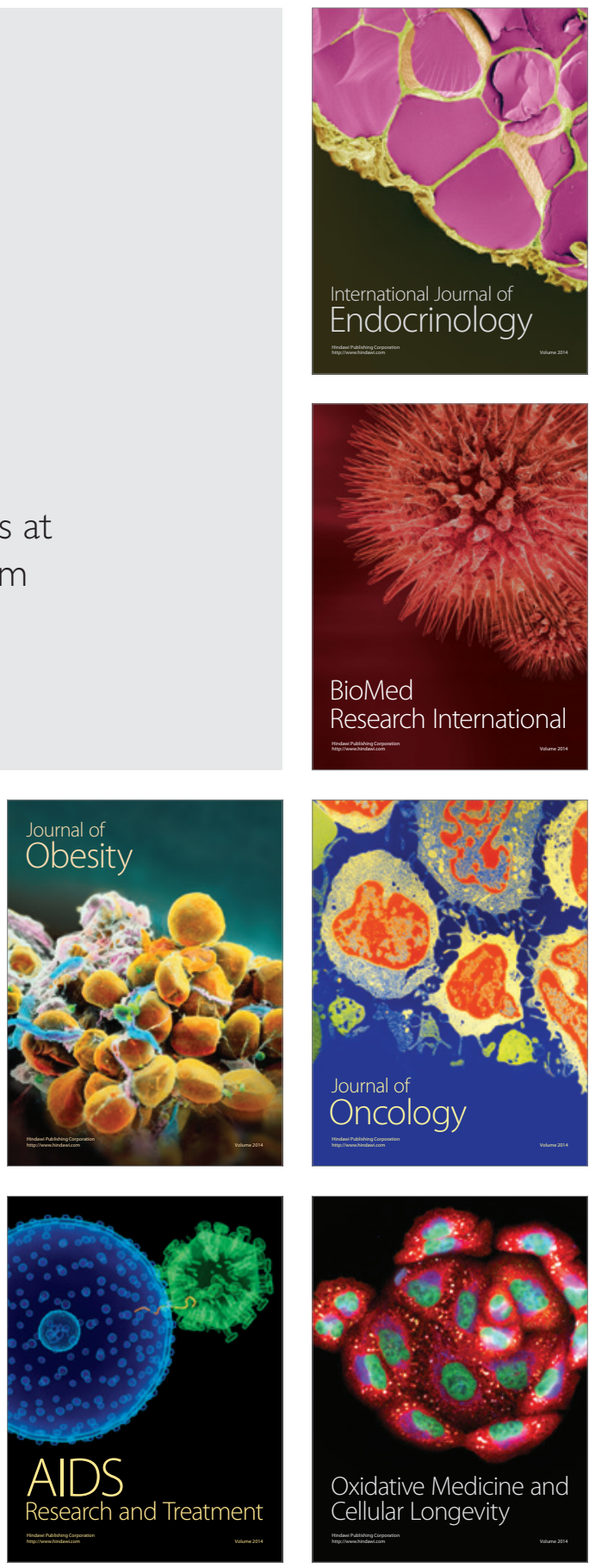